\title{
Real-world osimertinib for EGFR mutation-positive non-small-cell lung cancer with acquired T790M mutation
}

\author{
Fumio Imamura*,1, Madoka Kimura¹, Yukihiro Yano², Masahide Mori², Hidekazu Suzuki , \\ Tomonori Hirashima ${ }^{3}$, Shouichi Ihara ${ }^{4}$, Kiyoshi Komuta ${ }^{4}$, Takayuki Shiroyama ${ }^{5}$, Izumi \\ Nagatomo ${ }^{5}$ \& Toru Kumagai ${ }^{1}$ \\ ${ }^{1}$ Department of Thoracic Oncology, Osaka International Cancer Institute, 3-1-69 Otemae, Chuo-ku, Osaka, 541-8567, Japan \\ ${ }^{2}$ Department of Thoracic Oncology, National Hospital Organization Osaka Toneyama Medical Center, 5-1-1 Toneyama, Toyonaka, \\ Osaka, 560-8552, Japan \\ ${ }^{3}$ Department of Thoracic Oncology, Osaka Habikino Medical Center, 3-7-1 Habikino, Habikino, Osaka, 583-8588, Japan \\ ${ }^{4}$ Department of Respirology, Osaka Police Hospital, 2-6-40 Karasugatsuji, Tenoji-ku, Osaka, 543-8922, Japan \\ ${ }^{5}$ Department of Respiratory Medicine \& Clinical Immunology, Graduate School of Medicine, Osaka University, 2-2 Yamadaoka, \\ Suita, Osaka, 565-0871, Japan \\ *Author for correspondence: fimam@d6.dion.ne.jp
}

\begin{abstract}
Aim: Osimertinib is a key drug for EGFR mutation-positive non-small-cell lung cancer (NSCLC). As the hazards ratio of overall survival in comparison with first-generation EGFR-tyrosine kinase inhibitors was almost similar between FLAURA and ARCHER 1050, salvage use of osimertinib is still a treatment option. Patients \& methods: We retrospectively analyzed the clinical courses of EGFR mutation-positive NSCLC patients who were potential candidates for salvage osimertinib. Results: Among 524 patients enrolled from five hospitals, 302 patients underwent biopsy, with 52.6\% detection rate of T790M. Osimertinib was administered in $93.6 \%$ of the T790M-positive patients. The overall response rate and median progressionfree survival time of osimertinib were calculated with 147 patients, to be $55.6 \%$ and 17.2 months, respectively. Conclusion: Osimertinib is active for T790M-driven acquired resistance in EGFR-mutant NSCLC, but the detection of T790M was unsatisfactory.

Clinical Trial Registration: UMIN000028989 (UMIN Clinical Trials Registry)
\end{abstract}

First draft submitted: 10 March 2020; Accepted for publication: 28 April 2020; Published online: 14 July 2020

EGFR-tyrosine kinase inhibitors (EGFR-TKIs) are key drugs for treating EGFR-mutant non-small-cell lung cancer (NSCLC). Starting with gefitinib, several first-, second- and third-generation (1st-, 2nd- and 3rd-G, respectively) EGFR-TKIs have been developed. The toxicity and efficacy of these three generations of EGFR-TKIs were compared in three randomized trials, LUX-Lung 7, ARCHER 1050 and FLAURA [1-3]. All three trials showed clinical benefits of 2nd- or 3rd-G EGFR-TKIs compared with 1st-G EGFR-TKIs. Whereas all studies have shown improvement in progression-free survival (PFS) by 2nd- or 3rd-G EGFR-TKIs, statistically significant improvement of overall survival (OS) was attained only with osimertinib in FLAURA. OS improvement was also observed with dacomitinib in ARCHER1050, but statistical analysis was not performed because of the adoption of the gatekeeping method.

Osimertinib is a 3rd-G EGFR-TKI that yields a durable response in patients with NSCLC harboring common EGFR mutations [3,4]. It is also effective for T790M-mediated acquired resistance (AR) to 1st- or 2nd-G EGFRTKIs [5]. It was first approved as a salvage drug for T790M-mediated AR and was made available in Japan from 25 May 2016. Its frontline application became popular worldwide since the FLAURA study showed that frontline therapy using osimertinib improved PFS compared with 1st-G EGFR-TKIs. When PFS is compared between LUX-Lung 7, ARCHER 1050 and FLAURA, osimertinib appears to provide the longest PFS and is therefore one of the key drugs. It also has excellent penetrative capacity to the central nervous system. On the other hand, OS improvement is similar in frontline use between 2nd- and 3rd-G EGFR-TKIs, with hazard ratios of 0.760 in ARCHER1050 and 0.788 in FLAURA. Additionally, salvage osimertinib is a reasonable choice for the appreciable number of EGFR-mutant patients who started the treatment with 1st- or 2nd-G EGFR-TKIs but have not yet received a 3rd-G EGFR-TKI therapy. Therefore, there are two main first-line strategies for advanced $E G F R$-mutant

Future $\because$ Medicine 
NSCLC at present, one being frontline osimertinib and the other salvage osimertinib for T790M-positive AR preceded by frontline 1st- or 2nd-G EGFR-TKI therapy. A key future strategy using EGFR-TKIs would involve regulating the development of AR. Importantly, the mechanisms of AR to osimertinib differ between frontline and salvage therapy [6,7]. It must be said that the optimal usage of EGFR-TKIs is still under consideration.

Salvage osimertinib is presently applicable only for T790M-positive NSCLC. T790M is detected by rebiopsy of cancer tissue or by liquid biopsy of plasma. The reported rates of T790M in AR to 1st- or 2nd-G EGFR-TKIs have a wide distribution depending on the patient population examined and the platform for analysis adopted [8-11]. Pooled analysis revealed that T790M was detected in approximately 50-60\% of NSCLC cases with common EGFR mutations exhibiting AR to 1st- or 2nd-G EGFR-TKIs [12]. Because tissue biopsy is invasive and the sensitivity of liquid biopsy is not satisfactory, biopsy still presents a challenge especially in the real-world setting [13-15]. Therefore, it is important to know how frequently salvage osimertinib is applicable and how effective it is in a real-world setting. In the present study, patients from five Japanese institutes who had EGFR-mutant NSCLC and were potential candidates for salvage osimertinib were registered for retrospective analysis of their clinical courses.

\section{Patients}

Inclusion \& exclusion criteria

Eligible patients were required to be histologically or cytologically confirmed NSCLC harboring an activated EGFR mutation. Patients with disease recurrence after treatments with curative intent such as surgery or radiation therapy were included. In Japan, osimertinib in salvage use was approved on 25 May 2016. In order to accumulate the candidates for salvage osimertinib efficiently, eligible patients were required to meet one of the following two criteria:

- They were on any systemic treatment on 25 May 2016 and were considered to be in good conditions on 25 May 2016 enough to receive the next line of systemic treatment if the disease recurs in the future;

- They started first-line systemic treatment after 25 May 2016. Patients who had received a 3rd-G EGFR-TKI including osimertinib as the 1st EGFR-TKI or those with insufficient clinical records were excluded from the analysis.

\section{Patient subsets}

As mentioned above, osimertinib in salvage use was approved on 25 May 2016 in Japan. This means that the patients who had started the treatment with EGFR-TKIs before and after 25 May 2016 had different accessibility to osimertinib. Therefore, the patients in this study were divided into two groups, group 1 and group 2, consisting of patients who started any systemic treatments for EGFR-mutant NSCLC before and after 25 May 2016, respectively.

\section{Methods}

This is a protocol-based retrospective study. The study protocol (UMIN000028989) was approved by the ethics committee of each of the five participating institutes. The clinical data of patients with advanced NSCLC who met the eligibility criteria were retrospectively collected from each institute from 1 March 2017 to 31 October 2018 by an independent site-monitoring organization (EP-SOGO Co., Tokyo, Japan). Treatment responses were evaluated according to RECIST criteria version 1.1 [16]. A statistical analysis of response data was performed using the $\chi^{2}$ test. PFS of the patients who received osimertinib was calculated: PFS was defined as the time interval from the start of osimertinib to patient death or the detection of progressive disease (PD) to osimertinib. Kaplan-Meyer analysis and Cox proportional hazards tests were performed using SPSS statistics software, version 17.0. Patient accrual stopped on 31 October 2018 and the first data cut-off time was 31 October 2018. EGFR mutations in rebiopsy and liquid biopsy were examined by the tests which are reimbursed by insurance in Japan such as the clamp and covas method, not next-generation sequencing (NGS)-based methods.

\section{Results}

In total, 542 patients were enrolled between 25 January 2018 and 11 September 2018. Among these, 17 were excluded from the analysis because 1st- or 2nd-G EGFR-TKIs were switched to osimertinib without PD mainly due to toxicity. Patient characteristics of the 525 patients included are shown in Table 1. All patients except five with adenosquamous carcinoma carried adenocarcinoma; $0.8 \%$ patients had an ECOG performance status (PS) of 3; $95.4 \%$ had common EGFR mutations; $35.2 \%$ had undergone treatment with curative intent such as surgery or 
Table 1. Patient characteristics.

\begin{tabular}{|c|c|c|}
\hline Patients & Total enrolled & Osimertinib treated \\
\hline Number of patients & 525 & 147 \\
\hline Age (years), mean (range) & $67.0(35-89)$ & $66.7(38-87)$ \\
\hline \multicolumn{3}{|l|}{ Sex: } \\
\hline - Women & 351 & 97 \\
\hline - Men & 174 & 50 \\
\hline \multicolumn{3}{|l|}{ PS: } \\
\hline-0 & 229 & 33 \\
\hline-1 & 258 & 91 \\
\hline-2 & 31 & 17 \\
\hline-3 & 4 & 4 \\
\hline - Unknown & 3 & 2 \\
\hline \multicolumn{3}{|l|}{$E G F R^{\dagger}:$} \\
\hline - Exon19 del & 266 & 89 \\
\hline$-\mathrm{L} 858 \mathrm{R}$ & 235 & 58 \\
\hline - Minor mutation & 24 & - \\
\hline \multicolumn{3}{|l|}{ Stage: } \\
\hline - Recurrence $\ddagger$ & 185 & 45 \\
\hline - Stage I-III & 39 & 8 \\
\hline - Stage IV & 301 & 94 \\
\hline \multicolumn{3}{|l|}{ Group I: } \\
\hline-1 & 299 & 101 \\
\hline-2 & 226 & 46 \\
\hline \multicolumn{3}{|c|}{ 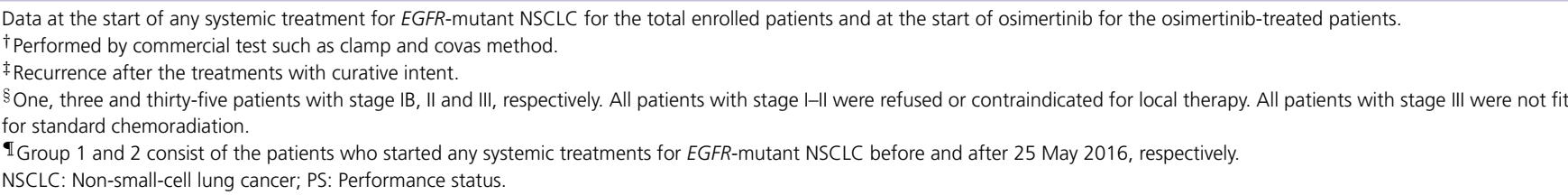 } \\
\hline
\end{tabular}

radiation. The consort diagram of the 525 patients is shown in Figure 1. Because the treatments prior to osimertinib were still effective in $132(25.1 \%$ of 525$)$ patients at the time of the data cut-off and these patients were therefore not administered osimertinib, the analysis of salvage osimertinib was performed on the remaining 393 patients.

Biopsy to detect T790M was performed using tissue (rebiopsy) or plasma (liquid biopsy) in 302 (76.8\%) of 393 patients. The repetition of biopsy and T790M positivity rates in rebiopsy and liquid biopsy are shown in Table 2 . Target lesions in the first to the third rebiopsy were intrathoracic $60 \%$, pleural effusion $19 \%$ and metastatic site $21 \%$, respectively. The cumulative positive rate of T790M was $52.6 \%$ (159 out of 302 patients). T790M was detected in $112(37.1 \%)$ patients in the first biopsy, indicating that repetition of biopsy is useful in detecting the mutation. A total of 462 biopsies was performed and the detection rate of T790M was $34.4 \%$ per biopsy ( 159 out of 462). The first three biopsies occupied approximately $96.3 \%$ of total biopsies, which in turn detected $98.1 \%$ of T790M-positive cases. Liquid biopsy was approved in December 2016 in Japan and was applied to $24.5 \%$ of all biopsies, with a T790M detection rate of $28.8 \%$.

Excluding two patients with uncommon EGFEventR mutations, 147 (37.4\%) out of 393 patients $(93.6 \%$ of 157 T790M-positive patients) received salvage osimertinib. On the other hand, the treatments for the patients for whom T790M was not detected were different depending on various conditions including treatment lines, age and PS of the patients. For example, second-line therapy for the patients who had undergone first-line therapy with an EGFR-TKI and developed T790M-unrelated AR were cytotoxic monotherapy (15.5\%), platinum doublets (46.5\%) and EGR-TKI-based therapy (38.0\%).

The adverse events (AEs) of osimertinib are summarized in Table 3. Discontinuation and grade $\geq 3$ AEs of osimertinib were observed in 8.2 and $10.2 \%$ of patients, respectively. Interstitial pneumonitis occurred in $2.0 \%$, leading to one treatment-related death. Responses were evaluable in 147 patients (101 in group 1 and 46 in group 2). The objective response rate (ORR), disease control rate (DCR) and median PFS of osimertinib were 55.6\%, 


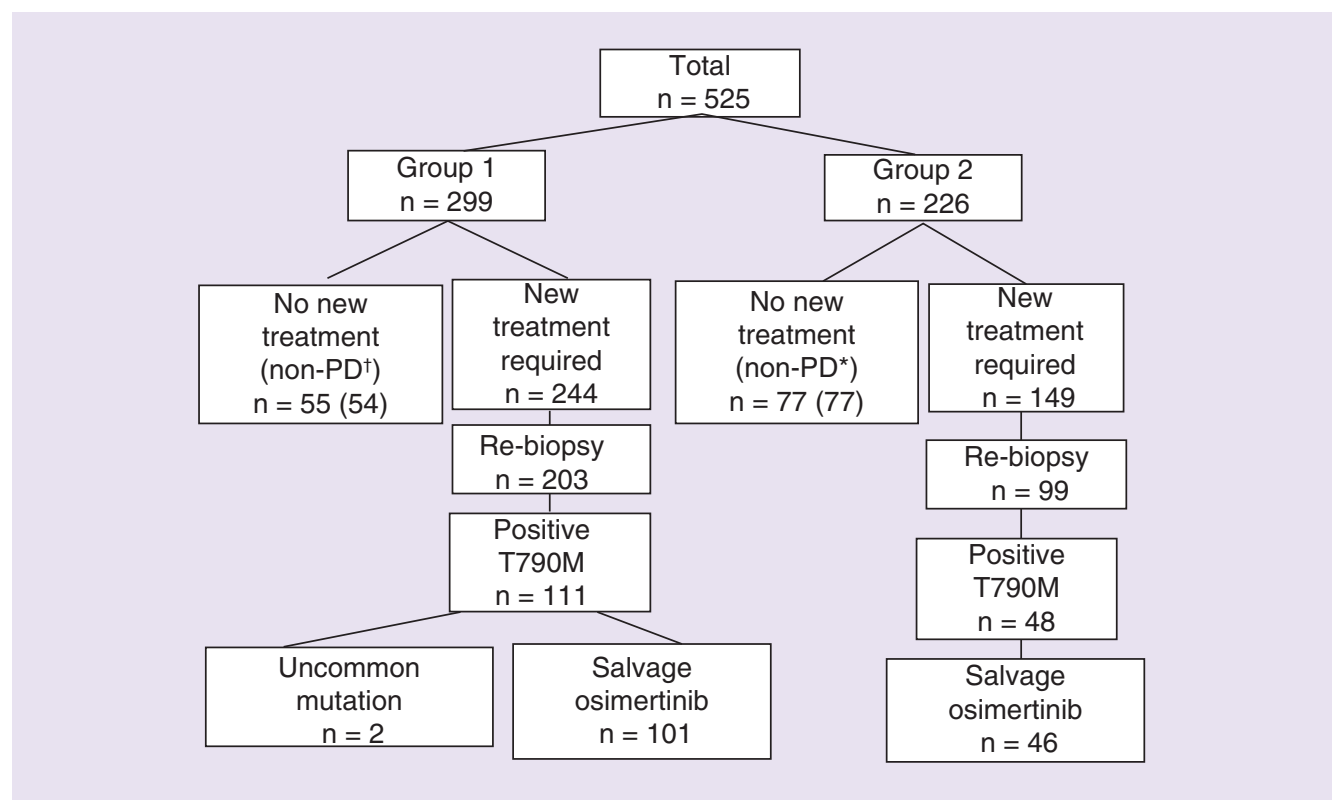

Figure 1. Consort diagram. Treatments for the patients whose non-small-cell lung cancer carried T790M but was not treated with osimertinib were as follows: 4 , continuation of the most recent treatment; 6 , no treatments; 1 , not specified.

†Osimertinib was not yet administered because the preceding treatments were still active at the data cut-off time. PD: Progressive disease.

\begin{tabular}{|c|c|c|c|c|c|c|}
\hline \multirow[t]{2}{*}{ Biopsy to detect T790M (\%) } & \multicolumn{2}{|c|}{ Total biopsy } & \multicolumn{2}{|c|}{ Rebiopsy $\S$} & \multicolumn{2}{|c|}{ Liquid biopsy } \\
\hline & $\mathrm{n}$ & T790M+ (\%) & $\mathrm{n}(\%)^{\dagger}$ & T790M+ (\%) & $\mathrm{n}(\%)^{\dagger}$ & $\mathrm{T790M}+(\%)$ \\
\hline $1 \mathrm{st}$ & 302 & $112(37.1)$ & 215 (71.2) & $88(40.9)$ & $87(28.8)$ & $24(27.6)$ \\
\hline 2nd & 110 & $33(30.0)$ & $82(74.5)$ & $23(28.0)$ & $28(25.5)$ & $10(35.7)$ \\
\hline $3 r d$ & 33 & $10(30.3)$ & $22(66.7)$ & $8(36.4)$ & $11(33.3)$ & $2(18.2)$ \\
\hline 4th-7th & 17 & $4(23.5)$ & $11(64.7)$ & $2(18.2)$ & $6(35.3)$ & $2(33.3)$ \\
\hline Overall: & 302 & $159(52.6)$ & & & & \\
\hline - Group 1 & 203 & $111(54.7)$ & & & & \\
\hline - Group 2 & 99 & $48(48.5)$ & & & & \\
\hline Total biopsy: & 461 & $159(34.5)$ & & & & \\
\hline - Rebiopsy & 329 & $121(36.8)$ & & & & \\
\hline - Liquid biopsy & 132 & $38(28.8)$ & & & & \\
\hline \multicolumn{7}{|c|}{$\begin{array}{l}\text { Biopsy includes rebiopsy with tissue and liquid biopsy with plasma. } \\
\text { †Numbers of rebiopsy or liquid biopsy/number of total biopsy. } \\
\text { ¥Positive rates of T790M in rebiopsy or liquid biopsy. } \\
\text { \& Target lesions in } 1 \text { st-3rd rebiopsy were intrathoracic } 60 \% \text {, pleural effusion } 19 \% \text { and metastatic site } 21 \% \text {, respectively. }\end{array}$} \\
\hline
\end{tabular}

88.9\% and 17.2 months, respectively (Table 4). No significant difference in PFS was observed with respect to age ( $<75$ vs $\geq 75$ ), sex, treatment line ( 2 nd vs 3 rd -4 th vs $\geq 5$ th) or method of biopsy (rebiopsy vs liquid biopsy) in univariate analysis (Table 5; Figures $2 \& 3$ ). On the other hand, significant prolongation of PFS was observed in patients with good PS (0 vs 1 vs $\geq 2$; Figure 2D). Patients with stage IV NSCLC and those in group 2 showed a tendency of shorter PFS. Multivariate analysis was performed adjusted with sex, type of EGFR mutations, age ( $<75$ vs $\geq 75$ ), PS ( 0 vs 1 vs $\geq 2$ ), stage (IV vs others), line of osimertinib treatment (2th vs 3 th- 4 th vs $\geq 5$ th), time of start of any systemic treatment (group 1 vs group 2), The analysis revealed that PS was the only factor that significantly correlated with longer PFS of salvage osimertinib (PS: 0 vs $1 ; p=0.002$; PS: $\geq 2$ vs $0 ; p=0.512$; overall; $\mathrm{p}=0.003)$. 


\section{Table 3. Adverse events.}

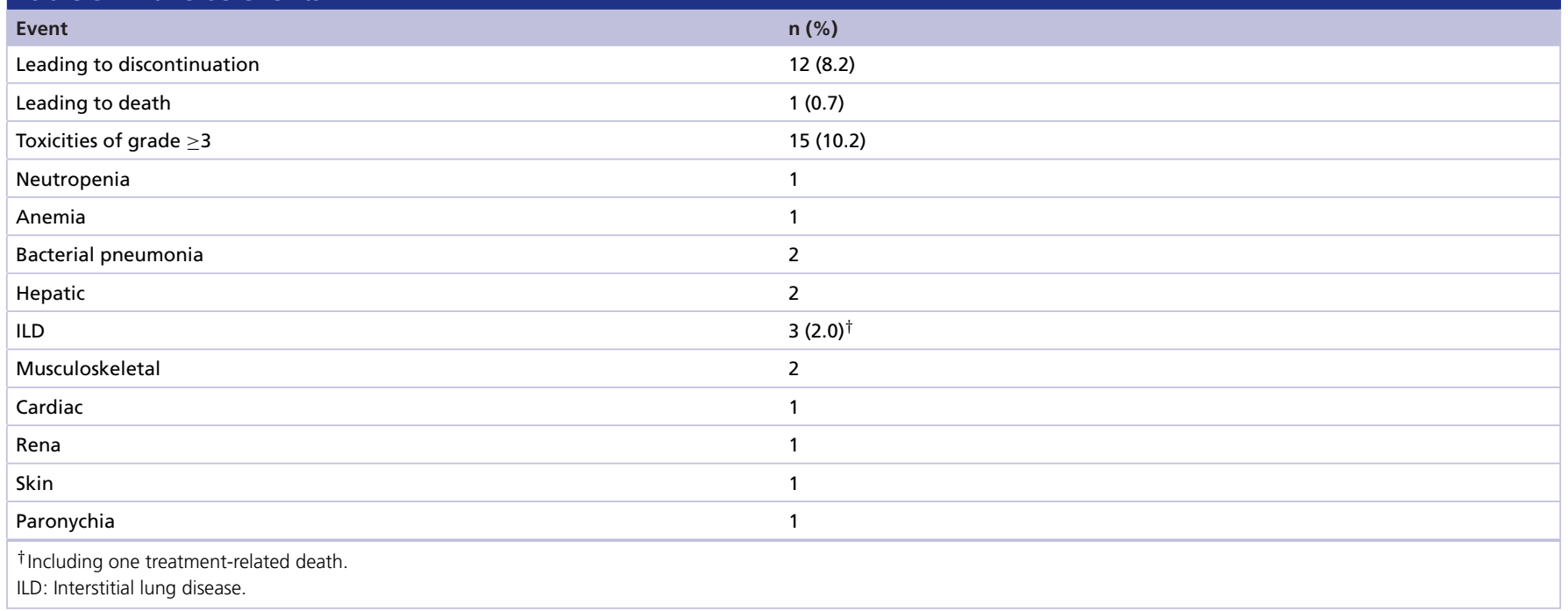

Table 4. Responses to osimertinib.

\begin{tabular}{|c|c|c|c|}
\hline Group & 1 & 2 & Total \\
\hline$C R$ & 2 & 1 & 3 \\
\hline PR & 52 & 16 & 68 \\
\hline SD & 27 & 14 & 41 \\
\hline PD & 7 & 7 & 14 \\
\hline $\mathrm{NE}$ & 13 & 8 & 21 \\
\hline $\mathrm{ORR}^{\dagger}$ & $61.4 \%$ & $44.7 \%$ & $56.3 \%$ \\
\hline
\end{tabular}

$\dagger_{p}=0.076$ by $\chi^{2}$ test.

CR: Compete response; DCR: Disease control rate; NE: Nonevaluable; ORR: Objective response rate; PD: Progressive disease; PR: Partial response; SD: Stable disease.

\section{Discussion}

This is one of the most comprehensive real-world studies to report the results of salvage osimertinib strategy for EGFR-mutant NSCLC. The detection of T790M mutation is a prerequisite for salvage osimertinib, thus resulting in limited application of the drug. At the time of the first data cut-off, $76.8 \%$ of our patients who had a history of AR to 1 st- or 2nd-G EGFR-TKIs underwent at least one rebiopsy or liquid biopsy and T790M was detected in 52.6\% of these. These biopsy results are comparable with those of a preceding Japanese report of 395 EGFR-mutated NSCLC patients from 28 centers, in which rebiopsy and T790M detection were successful in 79.5 and $46.8 \%$ cases, respectively [13]. Because T790M was detected in 50-60\% of patients with AR to 1 st- or 2nd-G EGFR-TKIs in a pooled analysis, these studies indicate that there may be little room for improvement of T790M detection in the real-world setting. Our data also suggest that liquid biopsy has a certain, but limited role in detecting T790M, which is consistent with previous reports $[17,18]$.

Reflecting real-world nature of this study, EGFR mutations in rebiopsy and liquid biopsy were examined by PCR-based tests which are reimbursed by insurance in Japan, not NGS-based methods. Therefore, information on changes of the genes other than EGFR were not available. In addition, because only one test which proved the presence of T790M mutation was necessary and sufficient for salvage osimertinib use, rebiopsy and liquid biopsy were performed as independent examinations. We have no data on concordance or discordance of the results of rebiopsy and liquid biopsy.

The toxicity of osimertinib was generally mild. As shown in Table 3, the frequency of grade $\geq 3$ AEs was $10.2 \%$, which is similar to the $12.2 \%$ reported in the Japanese subset of the AURA3 study [19,20]. When compared with 1st-G EGFR-TKI, osimertinib was reported to exhibit decreased severity in skin toxicity and transaminase elevation. On the contrary, a tendency of increased frequency was reported in the ejection fraction decrement and QT interval prolongation on electrocardiography [4]. ORR, DCR and PFS for osimertinib were reported to be 70.7, 


\begin{tabular}{|c|c|c|c|c|}
\hline Factors & $\mathrm{n}$ & Median $(95 \% \mathrm{Cl})$ & HR & p-value \\
\hline Overall & 147 & $17.2(11.9-22.5)$ & & \\
\hline \multicolumn{5}{|l|}{ Sex } \\
\hline $\operatorname{Men}^{\dagger}$ & 50 & $17.2(9.5-25.2)$ & & 0.88 \\
\hline Women & 97 & $18.7(12.2-25.2)$ & & 0.36 \\
\hline \multicolumn{5}{|l|}{ EGFR } \\
\hline $\mathrm{L}^{2} 858 \mathrm{R}^{\dagger}$ & 59 & $14.7(9.1-20.3)$ & & 0.89 \\
\hline 19del & 88 & $18.9(9.9-27.9)$ & & 0.65 \\
\hline \multicolumn{5}{|l|}{ Age (years) } \\
\hline$<75^{\dagger}$ & 78 & $15.5(7.6-23.3)$ & & 0.87 \\
\hline$>75$ & 69 & $19.7(12.8-26.7)$ & & 0.28 \\
\hline \multicolumn{5}{|l|}{ PS } \\
\hline 0 & 32 & $\mathrm{NR}^{\ddagger}$ & 0.36 & $<0.01$ \\
\hline$-1^{\dagger}$ & 93 & $10.9(8.1-15.7)$ & & \\
\hline$\geq 2$ & 22 & $18.7(3.3-34.1)$ & & 1.47 \\
\hline \multicolumn{5}{|l|}{ Stage } \\
\hline $\mathrm{IV}^{\dagger}$ & 94 & $13.6(6.9-20.4)$ & & 0.77 \\
\hline Others & 53 & $26.4(12.5-40,4)$ & & 0.06 \\
\hline \multicolumn{5}{|l|}{ Line } \\
\hline $2^{\dagger}$ & 55 & $15.5(9.2-21.7)$ & & 0.27 \\
\hline $3-4$ & 55 & $26.4(13.6-39.3)$ & & 0.83 \\
\hline$\geq 5$ & 37 & $10.9(6.8-15.1)$ & & 1.10 \\
\hline \multicolumn{5}{|c|}{ Group of patients } \\
\hline Group $1^{\dagger}$ & 101 & $18.9(13.4-24.4)$ & & 1.32 \\
\hline Group 2 & 46 & $10.6(7.5-13.8)$ & & 0.06 \\
\hline \multicolumn{5}{|c|}{ T790M detection with liquid biopsy or tissue biopsy } \\
\hline Tissue biopsy ${ }^{\dagger}$ & 111 & $17.2(9.9-15.8)$ & & 1.06 \\
\hline Liquid biopsy & 36 & $19.4(9.2-28.2)$ & & 0.74 \\
\hline \multicolumn{5}{|c|}{$\begin{array}{l}\text { †Control. } \\
\text { ¥Not reached. } \\
\text { HR: Hazard ratio; PS: Performance status. }\end{array}$} \\
\hline
\end{tabular}

95.1\% and $12.5 \mathrm{M}$, respectively, in the Japanese subset. In our study, the results of efficacy depended on the group of patients, as shown in Tables 4 and 5 and Figure 3B. The worse results in group- 2 patients may be ascribed to the inclusion of patients with poor conditions due to the real-world setting, while the better results in group-1 patients appear to reflect a selection bias. In fact, the median PFS was 11.5 months (95\% CI: 9.3-13.7 months) when group 2 patients with a PS of $0-1$, who had received osimertinib as the second or third line of therapy $(n=32)$, was analyzed. Group-1 patients had longer histories of the treatment with a preceding EGFR-TKI than group-2 patients and hence possibly included patients with relatively less-aggressive NSCLC or NSCLC controlled well by the preceding treatments. Prolonged PFS was consistently observed in patients with good PS both in univariate and multivariate analysis. Univariate analysis showed a tendency of longer PFS in patients with stage I-III NSCLC or with NSCLC that recurred after curative treatments. Notably, PFS was similar between the patients whose T790M was detected with rebiopsy and liquid biopsy.

Whereas prospective clinical trials are undoubtedly important to determine treatment strategies, they are limited in the extrapolation of results. In this sense, retrospective analysis has certain contribution. For example, Mu et al. reported real-world data of their recent experiences of salvage osimertinib in 91 patients [21]. DCR and PFS were reported to be $91.9 \%$ and 8.6 months for patients with detectable T790M mutation, respectively. The results mean that the data in AURA3 study can be almost reproduced in real-world setting. On the other hand, clinical practice for EGFR-mutant NSCLC involves many aspects, including first exposure to an EGFR-TKI, development of AR to it, rebiopsy or liquid biopsy, determination of the possible mechanism of AR such as T790M and treatment of the disease with AR. Even if osimertinib is highly effective against NSCLC with T790M-mediated AR, its low 
(A)

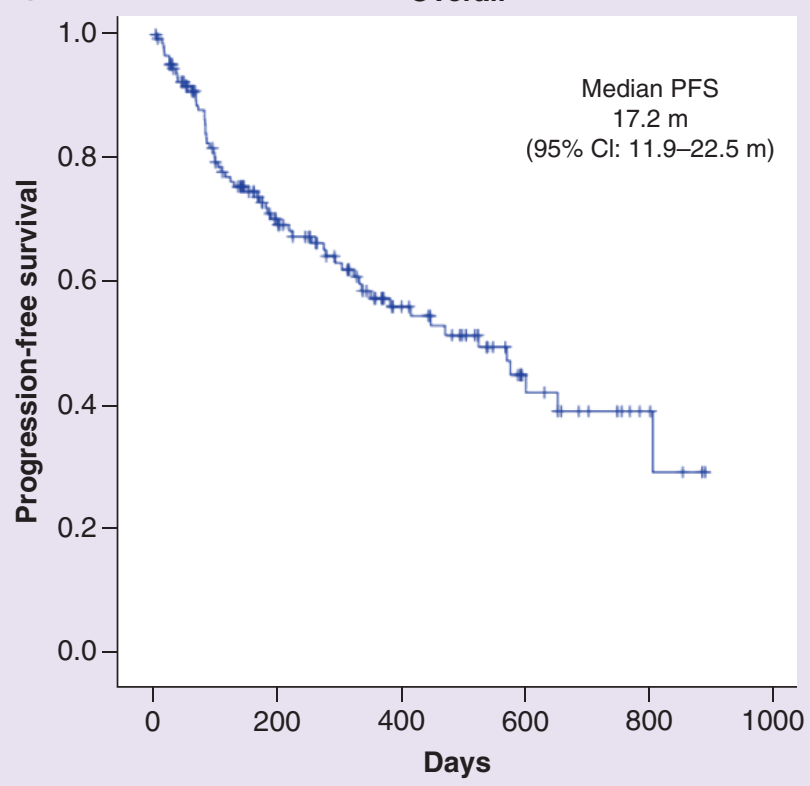

(C)

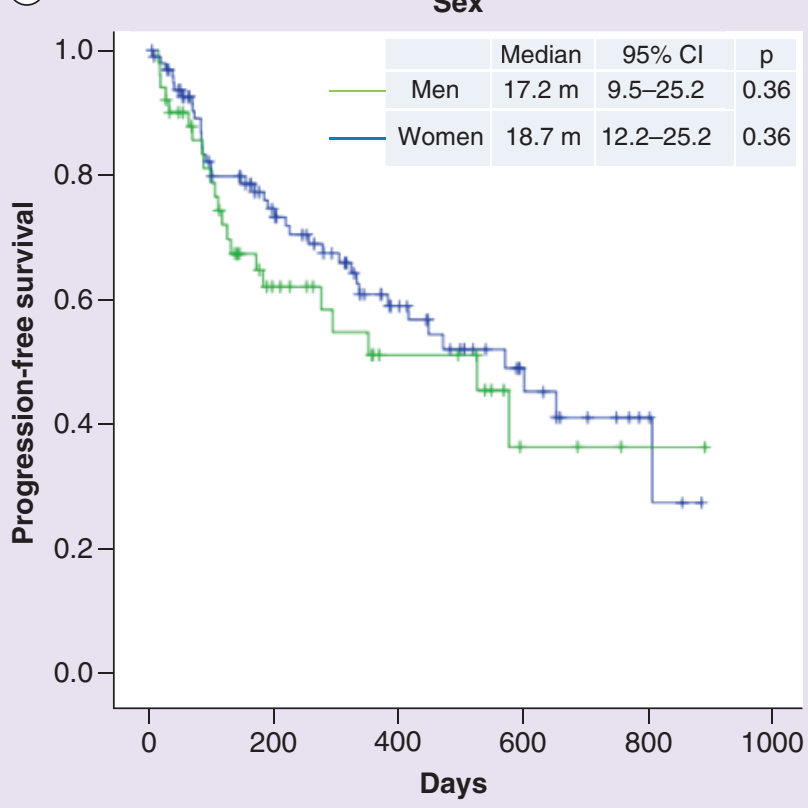

(B)

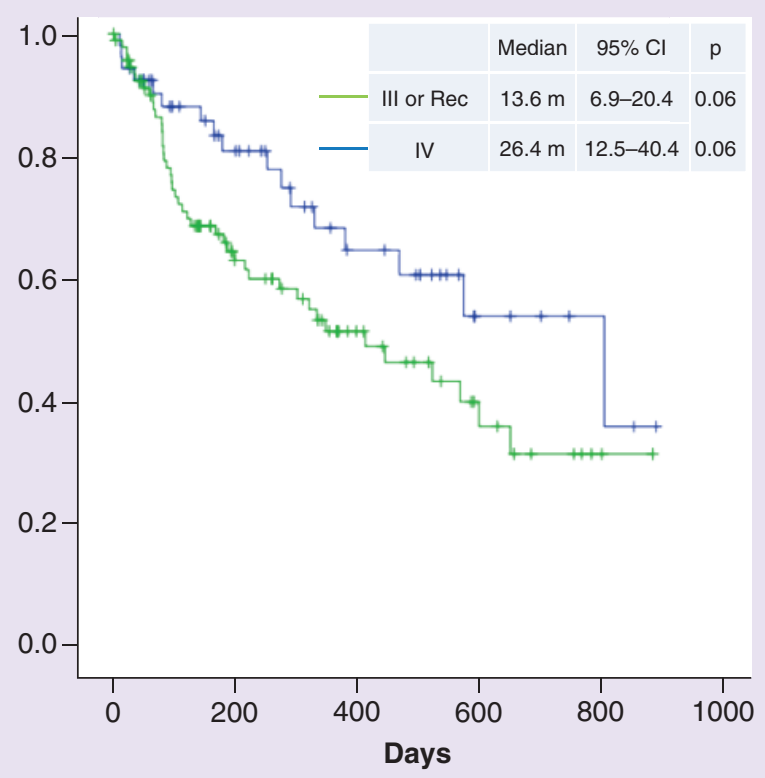

(D)
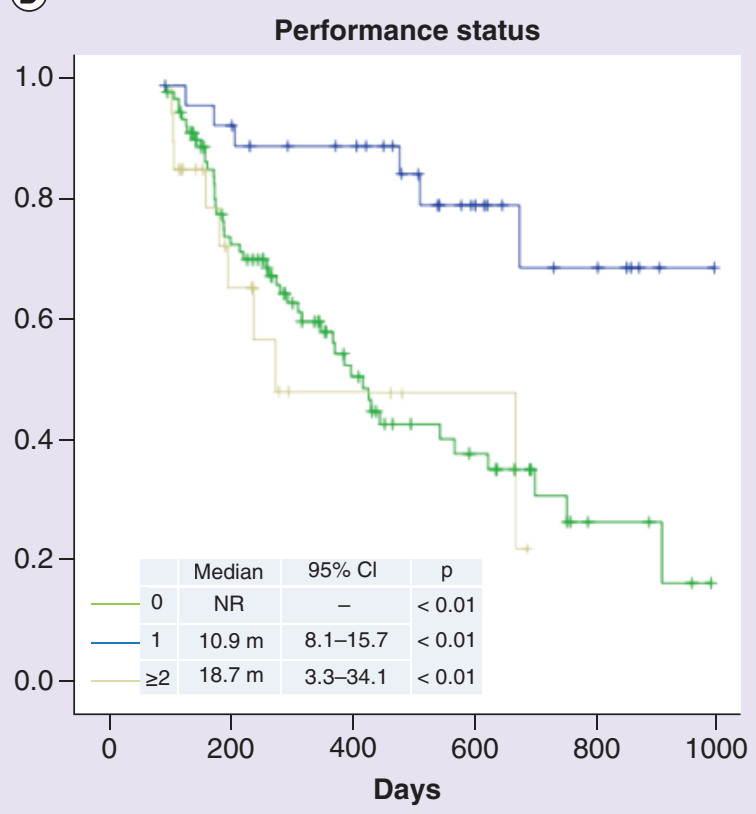

Figure 2. Progression-free survival. (A) Overall, (B) stage of disease at the start of osimertinib (recurrence after treatment with curative intent vs I-III vs IV), (C) sex (women vs men), (D) performance status at the start of osimertinib (0 vs 1 vs $\geq 2$ ). PFS: Performance free survival.

accessibility to osimertinib via poor detection of T790M, for example, must hamper its clinical utility. Thus, what is important is not only the efficacy of osimertinib, but also the frequency of success at each clinical step above, which we intended to evaluate in this study.

A comprehensive analysis of entire clinical processes is necessary to develop effective strategies for $E G F R$-mutant NSCLC. Recently, Zhou et al. comprehensively reported their experiences of 632 patients with EGFR-mutant NSCLC from a single institution [22]. According to their report, biopsy was performed in $55.5 \%$ of 555 patients who had experienced PD and T790M was detected in $43.5 \%$ of the biopsied patients (24.1\% of 555 patients). 3rd-G EGFR-TKIs were administered in 80 patients with T790M-positive NSCLC (14.4\% of 555 patients) and 

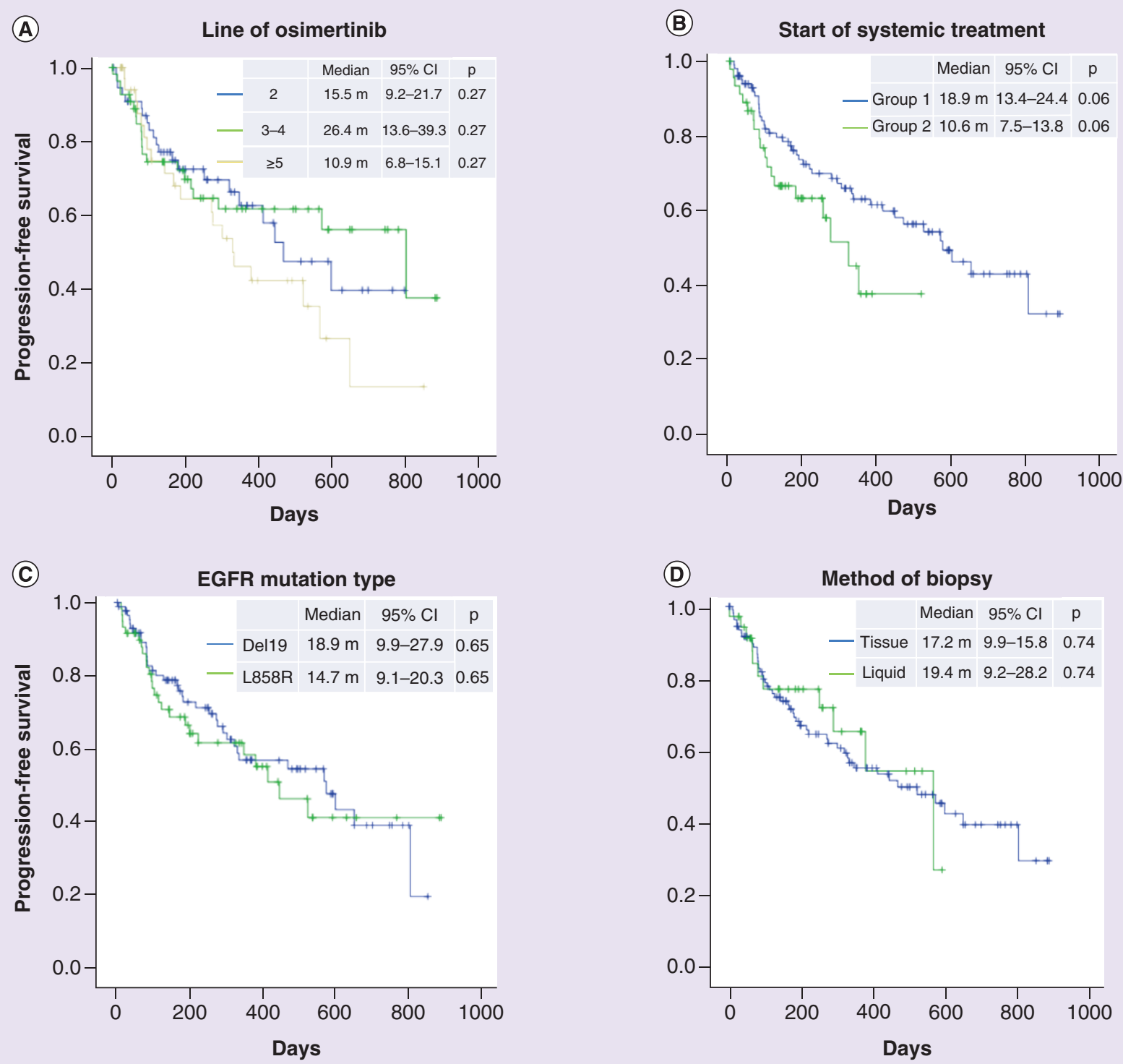

Figure 3. Progression-free survival (2). (A) Line of osimertinib (2nd vs 3rd-4th vs $\geq 5$ th), (B) start of any systemic treatment (before vs after 25 May 2016), (C) EGFR mutation type (exon 19 deletion vs L858R), (D) method of biopsy (rebiopsy vs liquid biopsy).

the PFS with 3rd-G EGFR-TKIs was 8.2 months. These results are fairly different from ours. Undoubtedly, these outcomes depend on the spread of rebiopsy and liquid biopsy, methods of T790M detection, characteristics of patients enrolled into studies and availability of 3rd-G EGFR-TKIs during study periods, among others. Their results, together with ours, indicate that salvage osimertinib has several problems to be solved.

Our study is limited by its retrospective nature and the fact that only Asian patients were included. However, it conveys the important message that the excellent performance of salvage osimertinib observed in the AURA3 study appears to be reproducible in a real-world setting. On the other hand, it also revealed that only $37.4 \%$ of the patients could receive osimertinib. Together with longer PFS, excellent CNS penetration and lower toxicity of osimertinib, these results indicate the advantage of frontline osimertinib. On the contrary, recent publication of the FLAURA study suggested that OS was comparable between 1st-G EGFR-TKIs and osimertinib in an Asian population and in patients with L858R-positive NSCLC. More effective strategies might appear in the future by combining different EGFR-TKIs or combining osimertinib with other categories of drugs. 


\title{
Future perspective
}

Molecular-targeted therapy has opened a new era of treatment for cancer, with EGFR-TKIs for NSCLC with activating EGFR mutations to be a representative. A notable feature of treatments for EGFR mutation-positive NSCLC is the availability of multiple TKIs. Although each EGFR-TKI has achieved remarkable success, there still exist challenges how to use these drugs over time. Our study showed that several clinically essential gates exist to deliver each regimen to a patient at the appropriate timing. This means that OS depends on the sequence of regimens and the ratio of passing through these gates. Therefore, not only the efficacy of each regimen but evaluation of entire treatment processes is essential in the future. Real-world studies are becoming increasingly important in the treatment for cancer with driver mutations.

\section{Summary points}

- Osimertinib, a third-generation EGFR-tyrosine kinase inhibitor (EGFR-TKI), is a key drug in the treatment of advanced non-small-cell lung cancer (NSCLC) harboring activated EGFR mutations and has attained the best hazard ratio of progression-free survival in comparison with first-generation EGFR-TKIs.

- At present, two strategies, frontline osimertinib and salvage osimertinib for the T790M-mediated progression to a first or second-generation EGFR-TKI, are popular in the treatment with EGFR-TKIs.

- Because hazard ratio of overall survival in comparison with first-generation EGFR-TKIs was almost similar between FLAURA study with osimertinib and ARCHER 1050 study with dacomitinib, salvage osimertinib may be a treatment option for certain subsets of the patients.

- Among 524 NSCLC patients with activated EGFR mutations in Japanese five hospitals, 393 patients experienced disease progression to a first- or second generation EGFR-TKI and 302 patients underwent rebiopsy or liquid biopsy.

- Rebiopsy and liquid biopsy detected T790M in 52.6\% of the patients tested; osimertinib was administered in $93.6 \%$ of the T790M-positive patients; overall response rate (ORR) and median progression-free survival time of osimertinib were $55.6 \%$ and 17.2 months, respectively.

- Activity of osimertinib for T790M-driven acquired resistance in EGFR-mutant NSCLC was confirmed in real world setting, but the probability of losing the opportunity to use osimertinib is quite high when salvage use of osimertinib is adopted.

\begin{abstract}
Author contributions
F Imamura performed planning of research, data analysis and preparation of manuscript M Kimura performed data collection and data analysis. Y Yano, H Suzuki, S Ihara and T Shiroyama performed data collection. M Mori, T Hirashima, I Nagatomo, K Komuta and T Kumagai performed planning of research and critical review of data.
\end{abstract}

\section{Acknowledgments}

The authors thank the patients and their families for making this study possible.

\section{Financial \& competing interests disclosure}

This research was supported by AstraZeneca. F Imamura reports honoraria from AstraZeneca, Chugai, Pfizer and Boehringer Ingelheim and research funds from AstraZeneca. T Hirashima reports honoraria and research funds from AstraZeneca, Chugai and Boehringer Ingelheim. M Mori reports speaker's fees from AstraZeneca, Chugai, Boehringer Ingelheim. I Nagatomo reports research grants and personal fees from AstraZeneca and Chugai. $Y$ Yano reports speaker's fees from AstraZeneca and Boehringer Ingelheim. H Suzuki reports personal fees from AstraZeneca and Chugai. T Kumagai reports speaker's fees from AstraZeneca, Boehringer Ingelheim, Pfizer and Chugai and research funds from Chugai, AstraZeneca, Pfizer and Boehringer Ingelheim. S Ihara, M Kimura and T Shiroyama report no conflict of interest. The authors have no other relevant affiliations or financial involvement with any organization or entity with a financial interest in or financial conflict with the subject matter or materials discussed in the manuscript apart from those disclosed.

We would like to thank Editage (www.editage.com) for editing the manuscript.

Ethical conduct of research

The authors have no other relevant affiliations or financial involvement with any organization or entity with a financial interest in or financial conflict with the subject matter or materials discussed in the manuscript apart from those disclosed. 
Open access

This work is licensed under the Attribution-NonCommercial-NoDerivatives 4.0 Unported License. To view a copy of this license, visit http://creativecommons.org/licenses/by-nc-nd/4.0/

\section{References}

Papers of special note have been highlighted as: $\bullet$ of interest

1. Park K, Tan EH, O’Byrne K et al. Afatinib versus gefitinib as first-line treatment of patients with EGFR mutation-positive non-small-cell lung cancer (LUX-Lung 7): a Phase 2B, open-label, randomised controlled trial. Lancet Oncol. 17(5), 577-589 (2016).

2. Wu YL, Cheng Y, Zhou X et al. Dacomitinib versus gefitinib as first-line treatment for patients with EGFR-mutation-positive non-small-cell lung cancer (ARCHER 1050): a randomised, open-label, Phase III trial. Lancet Oncol. 18(11), 1454-1466 (2017).

- A Phase III trial of second generation (dacomitinib) vs first-generation EGFR-tyrosine kinase inhibitor (EGFR-TKI), which showed improved progression-free survival and suggested better overall survival.

3. Soria JC, Ohe Y, Vansteenkiste J et al. Osimertinib in untreated EGFR-mutated advanced non-small-cell lung cancer. N. Engl. J. Med. 378(2), 113-125 (2018).

4. Ramalingam SS, Vansteenkiste J, Planchard D et al. Overall survival with osimertinib in untreated, EGFR-mutated advanced NSCLC. N. Engl. J. Med. 382, 41-50 (2020).

- A Phase III trial of osimertinib (third generation) vs first-generation EGFR-TKI, which showed improved progression-free survival and overall survival.

5. Mok TS, Wu YL, Ahn MJ et al. Osimertinib or platinum-pemetrexed in EGFR T790M-positive lung cancer. N. Engl. J. Med. 376(7), 629-640 (2017).

6. Leonetti A, Sharma S, Minari R et al. Resistance mechanisms to osimertinib in EGFR-mutated non-small-cell lung cancer. Br. J. Cancer 121(9), 725-737 (2019).

7. Gray JE, Okamoto I, Sriuranpong V et al. Tissue and Plasma EGFR mutation analysis in the FLAURA trial: osimertinib vs comparator EGFR-TKI as first-line treatment in patients with EGFR mutated advanced NSCLC. Clin. Cancer Res. 25(22), 6644-6652 (2019).

8. Yu HA, Arcila ME, Rekhtman N et al. Analysis of tumor specimens at the time of acquired resistance to EGFR-TKI therapy in 155 patients with EGFR-mutant lung cancers. Clin. Cancer Res. 19, 2240-2247 (2013).

9. Oxnard GR, Arcila ME, Sima CS et al. Acquired resistance to EGFR tyrosine kinase inhibitors in EGFR-mutant lung cancer: distinct natural history of patients with tumors harboring the T790M mutation. Clin. Cancer Res. 17, 1616-1622 (2017).

10. Cabanero M, Tsao MS. Circulating tumour DNA in EGFR-mutant non-small-cell lung cancer. Curr. Oncol. 25(Suppl. 1), S38-S44 (2018).

11. Li X, Zhou C. Comparison of cross-platform technologies for EGFR T790M testing in patients with non-small-cell lung cancer. Oncotarget 8(59), 100801-100818 (2017).

12. Wang ZF, Ren SX, Li W, Gao GH. Frequency of the acquired resistant mutation T790 M in non-small-cell lung cancer patients with active exon 19Del and exon 21 L858R: a systematic review and meta-analysis. BMC Cancer. 18(1), 148 (2018).

13. Nosaki K, Satouchi M, Kurata $\mathrm{T}$ et al. Rebiopsy status among non-small-cell lung cancer patients in Japan: a retrospective study. Lung Cancer 101, 1-8 (2016).

- Shows real-world efficacy of rebiopsy in detecting T790M mutation in EGFR-mutant non-small-cell lung cancer.

14. Zhou J, Zhao C, Zhao J et al. Rebiopsy and liquid biopsy for patients with non-small-cell lung cancer after EGFR-tyrosine kinase inhibitor failure. Thorac. Cancer 10(4), 957-965 (2019).

15. Hotta K, Ninomiya K, Ichihara E et al. Significance of rebiopsy of histological tumor samples in advanced non-small-cell lung cancer in clinical practice. Int. J. Clin. Oncol. 24(1), 41-45 (2019).

16. Eisenhauer EA, Therasse P, Bogaerts J et al. New response evaluation criteria in solid tumours: revised RECIST guideline (version 1.1). Eur. J. Cancer 45(2), 228-247 (2009).

17. Oellerich M, Christenson RH, Beck J et al. Plasma EGFR mutation testing in non-small-cell lung cancer: a value proposition. Clin. Chim. Acta 495, 481-486 (2019).

18. Saarenheimo J, Eigeliene $\mathrm{N}$ andersen $\mathrm{H}$ et al. The value of liquid biopsies for guiding therapy decisions in non-small-cell lung cancer. Front. Oncol. 9, 129 (2019).

19. Akamatsu H, Katakami N, Okamoto I et al. Osimertinib in Japanese patients with EGFR T790M mutation-positive advanced non-small-cell lung cancer: AURA3 trial. Cancer Sci. 109(6), 1930-1938 (2018).

- Toxicity and efficacy of salvage osimertinib in Japanese subset in AURA3 study, which corresponds to the patients in our study.

20. Hirashima T, Satouchi M, Hida T et al. Osimertinib for Japanese patients with T790M-positive advanced non-small-cell lung cancer: a pooled subgroup analysis. Cancer Sci. 110(9), 2884-2893 (2019).

21. Mu Y, Xing P, Hao X et al. Real-world data of osimertinib in patients with pretreated non-small-cell lung cancer: a retrospective study. Cancer Manag. Res. 11, 9243-9251 (2019).

22. Zhou J, Zhao C, Zhao J et al. Rebiopsy and liquid biopsy for patients with non-small-cell lung cancer after EGFR-tyrosine kinase inhibitor failure. Thorac. Cancer. 10(4), 957-965 (2019). 